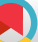

\title{
Prealbumin May Predict Clinical Outcomes in Children with Severe Mycoplasma pneumoniae Pneumonia
}

\author{
Cheng-Yi Wang (iD) ${ }^{1,2,}{ }^{*}$, Chao-Min Song ${ }^{3}$, Shi-Biao Wang ${ }^{4}$ and Guang-Hua Liu (iD ${ }^{1, * *}$ \\ ${ }^{1}$ Department of Pediatrics, Fujian Provincial Maternity and Children's Hospital, Affiliated Hospital of Fujian Medical University, Fuzhou, China \\ ${ }^{2}$ Engineering Research Center for Medical Data Mining and Application of Fujian Province, Xiamen, China \\ ${ }^{3}$ Department of Neonatology, Fujian Provincial Maternity and Children's Hospital, Affiliated Hospital of Fujian Medical University, Fuzhou, China \\ ${ }^{4}$ Pediatric Intensive Care Unit, Fujian Provincial Maternity and Children's Hospital, Affiliated Hospital of Fujian Medical University, Fuzhou, China \\ "Corresponding author: Department of Pediatrics, Fujian Provincial Maternity and Children's Hospital, Affiliated Hospital of Fujian Medical University, Fuzhou 350001, China. \\ Email: wangchengyi79@126.com \\ "Corresponding author: Department of Pediatrics, Fujian Provincial Maternity and Children's Hospital, Affiliated Hospital of Fujian Medical University, Fuzhou 350001, China. \\ Email: 20145119@qq.com
}

Received 2019 September 01; Revised 2019 October 21; Accepted 2019 November 09.

\begin{abstract}
Background: Serum prealbumin (PA) levels have been found to have prognostic value in patients with infectious diseases.

Objectives: We aimed to determine the relationship between PA levels and clinical outcomes in children with severe Mycoplasma pneumoniae pneumonia (MPP).

Methods: We retrospectively reviewed the data of 163 children with severe MPP who were treated in our hospital from January 2015 to December 2017. The demographic and clinical data of the subjects were collected and statistically analyzed.

Results: Our cohort was divided into quartiles by PA level. The average D-dimer level, pediatric risk of mortality (PRISM) III score, total length of stay in hospital (TLSH), C-reactive protein/PA ratio, and proportion of patients with invasive intubation significantly decreased as the serum PA level increased. In addition, the PRISM III estimated mortality and the procalcitonin/PA ratio significantly differed among the four PA groups. Binary logistic regression analysis showed that PA level (odds ratio [OR]: 0.846, 95\% confidence interval $[\mathrm{CI}]: 0.773-0.926, \mathrm{P}=0.000$ ) and invasive intubation (OR: 4.581, 95\% CI:1.730 -12.124, P=0.002) were independently correlated with PRISM III scores. Multiple linear regression analysis showed that PA level $(\beta=-0.025,95 \% \mathrm{CI}:-0.048$ to $-0.002, \mathrm{P}=0.032)$ was negatively associated with the normal score of TLSH calculated using the Blom formula.

Conclusions: The present study demonstrated that in children with severe MPP, low PA levels correlated with increased PRISM III scores and prolonged TLSH, suggesting that low PA levels contribute to the progression of severe MPP and possibly lead to poor outcomes.
\end{abstract}

Keywords: Mycoplasma pneumoniae, Severity, Prealbumin, PRISM III, Retrospective, China

\section{Background}

The incidence of severe Mycoplasma pneumoniae pneumonia (MPP) has recently increased (1). Severe MPP may be caused by infection with drug-resistant M. pneumoniae. In children, severe MPP is associated with mixed infection and extrapulmonary complications such as optic neuritis, acute kidney injury, and secondary hemolytic anemia (2$6)$. In China, MPP is a leading cause of death in children (7). Thus, MPP not only causes great economic loss but also has a lasting social impact on families, schools, and even on society as a whole (8). Therefore, it is necessary to find an objective biomarker that can predict progression to severe MPP early in the course of the disease in order to improve prognosis.

Prealbumin (PA) is a tetrameric unglycosylated plasma protein synthesized in the liver and is an indicator of the nutritional and inflammatory status (9). As PA has a shorter half-life (average, $\sim 2$ days) than albumin, it can more precisely indicate the nutritional and inflammatory status of a patient at the moment of measurement and has garnered considerable clinical attention. A prospective observational cohort study showed that low PA levels are common (36\%) among patients with acute heart failure, and are associated with higher short-term mortality (10). A single-center, retrospectively conducted from November 2003 to March 2016 showed that preoperative serum PA levels predicted patient outcomes after continuous-flow left ventricular assist device implantation (11). A crosssectional study of 867 patients with acute coronary syndrome showed that PA was negatively and independently 
associated with angiographic severity, indicating its potential as a biomarker for estimating the burden of coronary atherosclerosis (12). Roche et al. also reported that patients with preoperative PA values below the normal reference range had an increased risk of postoperative wound complications following total knee arthroplasty (13). However, to the best of our knowledge, there has been little research focusing on PA levels in children with severe MPP. Therefore, this retrospective study was conducted to test the hypothesis that PA values correlate with the severity and progression of MPP in pediatric patients.

\section{Objectives}

This retrospective descriptive study was conducted from January 2015 to December 2017, on children with severe MPP admitted to a tertiary hospital in Fujian province, China. The diagnosis of pneumonia was based on the clinical manifestations, which included dry or productive cough, fever, dyspnea, abnormal breathing sounds, and radiological pulmonary abnormalities. The diagnosis of $M$. pneumoniae infection was based on positive serological results: total antibody $\geq 1: 640$, four-fold or greater rise of M. pneumoniae -IgG and positive IgM antibody. Severe MPP was diagnosed when any one of the following criteria was met.

(1) Obvious tachypnea: respiratory rate $\geq 60$ breaths. $\min ^{-1}$ at age $<2$ months, $\geq 50$ breaths. min $^{-1}$ at ages 2 - 12 months, $\geq 40$ breaths. min $^{-1}$ at ages $1-5$ years, and $\geq 30$ breaths. min $^{-1}$ at age $\geq 5$ years (excluding those with fever and were crying), with or without dyspnea (defined as nasal alar breathing, groaning, and the three-concave sign) and cyanosis.

(2) Hypoxemia: pulse blood oxygen saturation $\leq 0.92$ under conditions of induced air.

(3) Continuous fever: axillary temperature $\geq 38.5^{\circ} \mathrm{C}$ or chest radiological progression after macrolide therapy for 7 days or longer.

(4) Invasion of many lobar and segmental bronchi or infiltration $\geq 2 / 3$ rd of a lung on chest X-ray examination.

(5) Pulmonary complications such as pleural effusion, atelectasis, pulmonary necrosis, or lung abscess.

(6) Evidence of severe damage to other organ systems such as central nervous system infection, heart failure, myocarditis, and obvious electrolyte or acid-base disturbance.

The exclusion criteria were abnormal airway development, congenital disease, inherited metabolic or autoimmune disease, neoplastic disease, malnutrition, or incomplete clinical data.

\section{Methods}

Medical records were reviewed for the following: (1) patient description, including age, gender, length of hospitalization, requirement for supplemental oxygen, and length of fever; (2) findings of investigative workup done within $24 \mathrm{~h}$ of hospital admission, such as white blood cell (WBC) count, platelet (PLT) count, hemoglobin (Hb), red blood cell volume distribution width (RDW), C-reactive protein (CRP), procalcitonin (PCT), PA, lactate dehydrogenase (LDH), MB isoenzyme of creatine kinase (CK-MB), alanine aminotransferase (ALT), and D-dimer level measurements, and blood culture, electrocardiography, and chest radiography; (3) disease severity parameters, including systolic blood pressure, temperature, mental status, heart rate, pupillary reflexes, acidosis $(\mathrm{pH})$, total $\mathrm{CO}_{2}, \mathrm{PCO}_{2}$, arterial $\mathrm{PaO}_{2}$, glucose, potassium, creatinine, urea, WBC count, prothrombin time or partial thromboplastin time, and PLT count. The worst values of the aforementioned diseaseseverity parameters within $24 \mathrm{~h}$ after admission were used to generate the pediatric risk of mortality (PRISM) III score. Coinfection detected within $24 \mathrm{~h}$ after admission was recorded using the following techniques: blood culture for bacterial infection, passive agglutination method for the detection of M. pneumoniae antibody, indirect fluorescent antibody test for nine common viruses (respiratory syncytial virus, adenovirus, influenza viruses A and B, parainfluenza viruses 1, 2, and 3, Legionella pneumophila, Chlamydia pneumoniae, M. pneumoniae, and Coxiella burnetii), fluorometric PCR assay for the detection of EpsteinBarr virus and cytomegalovirus, colloidal gold method for the detection of enterovirus type 71, and enzyme-linked immunosorbent assay for the detection of Herpes simplex virus in serum samples.

The study was approved by the ethics committee of our hospital (no, 2017-042). All subjects and their family members signed informed consent forms, and the subjects' data were anonymized before analysis.

All data were analyzed using SPSS version 23.0 (IBM). The findings of descriptive analyses were reported as absolute frequencies or rates in the case of categorical variables, as medians (minimum-to-maximum values) in the case of quantitative variables with non-parametric distributions, and as mean \pm SD in the case of quantitative variables with normal distributions. Quantitative variables were compared among the study groups by using one-way analysis of variance (ANOVA) or the Kruskal-Wallis test, as appropriate. Categorical variables were compared using the $\chi^{2}$ test. Linear correlation analysis of statistically significant variables was performed. Based on the results of the linear correlation analysis, binary multivariate logistic regression analysis or multivariate linear regression anal- 
ysis was performed to identify independent variables predicting hospital mortality and the total length of stay in the hospital (TLSH). P values $<0.05$ were considered statistically significant.

\section{Results}

\subsection{Demographic Characteristics}

A total of 180 children with severe MPP were admitted to our hospital. In the subsequent data collection, seven children were excluded due to incomplete clinical data, three children were excluded due to malnutrition, three children were excluded due to congenital tracheal stenosis, two children were excluded due to methylmalonic acidemia, and two children were excluded due to acute lymphoblastic leukemia (Figure 1). Finally, our study included 163 infants with severe MPP, including 86 boys and 77 girls (male-to-female ratio, 1.1:1).

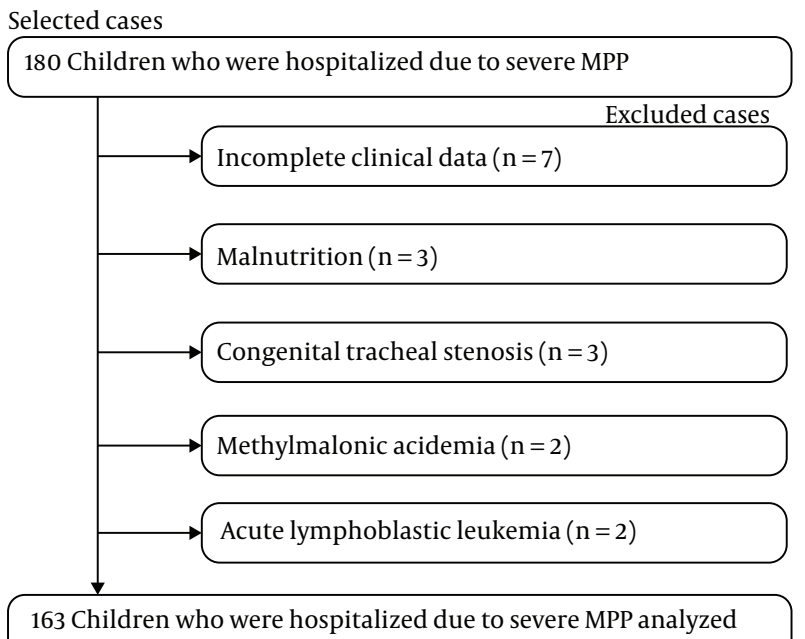

Figure 1. Flow chart of the study selection and exclusion process

We divided our cohort into quartiles by PA level as follows: first quartile, $\mathrm{PA} \leq 10.86 \mathrm{mg} / \mathrm{dL}$; second quartile, $10.86<\mathrm{PA} \leq 14.43 \mathrm{mg} / \mathrm{dL}$; third quartile, $14.43<\mathrm{PA} \leq 20.73$ $\mathrm{mg} / \mathrm{dL}$; and fourth quartile, PA $>20.73 \mathrm{mg} / \mathrm{dL}$. These four groups were similar with respect to gender and age $(\mathrm{P}>$ 0.05). PRISM III score and PRISM III-estimated mortality were non-linearly related to the other statistically significant variables. For the purpose of statistical analysis, we divided our cohort into two groups according to previously published methods (14) as follows: PRISM III score > p75 (n $=39)$ and PRISM III score $\leq$ p75 $(\mathrm{n}=124)$.

\subsection{Clinical Characteristics}

Fever was the most common symptom among the overall cohort, and the length of fever did not significantly differ among the PA quartiles. The proportion of patients with invasive intubation significantly increased with decreasing PA levels $(\mathrm{P}<0.05$; Table 1$)$. Coinfections and extrapulmonary complications were common among the children with MPP, but there were no significant differences between the PA quartiles in terms of the rates of coinfections, cardiovascular complications, gastrointestinal complications, neurological complications, hematological complications, extrapulmonary complications, or multisystem complications.

Regarding laboratory examinations, the average Ddimer level, PRISM III score, TLSH, and CRP/PA ratio significantly decreased as the serum PA level increased. In addition, the PRISM III estimated mortality and PCT/PA ratio significantly differed among the four groups $(\mathrm{P}<0.05)$. However, no differences were observed in terms of the WBC count, Hb level, PLT count, RDW, and the CRP, PCT, LDH, CKMB, and ALT levels (Table 1 ).

\subsection{Correlation Between Clinical Characteristics and PRISM III Scores}

The following clinical and laboratory parameters with statistically significant differences among the four PA groups were further analyzed using binary logistic regression analysis to identify variables that were independently associated with the PRISM III estimated mortality: invasive intubation (categorical variable) and D-dimer, PA, TLSH, and the CRP/PA and PCT/PA ratios (continuous variables). We found that PA level (odds ratio [OR]: 0.846, 95\% confidence interval $[\mathrm{CI}]$ : $0.773-0.926 ; \mathrm{P}=0.000)$ and invasive intubation (OR: 4.581, 95\% CI: 1.730 - 12.124; $\mathrm{P}=0.002$ ) were independent variables that entered the final model (Table 2). This suggested that lower PA levels increased the risk of hospital mortality independently of the effect of possible confounders.

\subsection{Correlation Between Clinical Characteristics and TLSH}

The following clinical and laboratory parameters with statistically significant differences among the PA groups were analyzed using multiple linear regression to identify variables independently associated with TLSH: invasive intubation (categorical variable) and D-dimer, PA, CRP/PA ratio, and PCT/PA ratio (continuous variables). The TLSH data were not normally distributed and were transformed using the Blom formula to achieve a normal distribution. The dependent variable was thus the normal score of TLSH as calculated using the Blom formula. In the multiple linear regression analysis model $(F=3.527, P=0.016)$, the 


\begin{tabular}{|c|c|c|c|c|c|c|c|}
\hline Characteristics & ALL & $\begin{array}{l}\text { First Quartile, PA } \\
\leq 10.86 \mathrm{mg} / \mathrm{dL}(\mathrm{N}= \\
49)\end{array}$ & $\begin{array}{l}\text { Second Quartile, } \\
10.86 \mathrm{mg} / \mathrm{dL}<\mathrm{PA} \\
\leq 14.43 \mathrm{mg} / \mathrm{dL}(\mathrm{N}= \\
33)\end{array}$ & $\begin{array}{c}\text { Third Quartile, } \\
14.43 \mathrm{mg} / \mathrm{dL}<\mathrm{PA} \\
\leq 20.73 \mathrm{mg} / \mathrm{dL}(\mathrm{N}= \\
\mathbf{4 8})\end{array}$ & $\begin{array}{l}\text { Fourth Quartile, } \\
\text { PA }>20.73 \mathrm{mg} / \mathrm{dL} \\
\quad(\mathrm{N}=33)\end{array}$ & $\mathbf{F} / \mathbf{Z} / \chi^{2}$ & P Value \\
\hline Length of fever, $d$ & $4.26(0-37.00)$ & $5.46(0-37.00)$ & $3.82(0-22.00)$ & $4.21(0-18.00)$ & $3.05(0-17.00)$ & 3.100 & 0.376 \\
\hline $\begin{array}{l}\text { Invasive } \\
\text { intubated (n, \%) }\end{array}$ & $35-21.5$ & $16-32.6$ & $9-27.3$ & $6-12.5$ & $4-12.1$ & 8.294 & 0.040 \\
\hline $\begin{array}{l}\text { Coinfection of two } \\
\text { or more noxaes (n, } \\
\%)\end{array}$ & $44-27.0$ & $14-28.6$ & $9-27.3$ & $11-22.9$ & $10-30.3$ & 0.651 & 0.885 \\
\hline $\begin{array}{l}\text { Extrapulmonary } \\
\text { complications of } \\
\text { two or more } \\
\text { systems }(n, \%)\end{array}$ & $33-20.2$ & $11-22.4$ & $6-18.1$ & $10-20.8$ & $6-18.1$ & 0.332 & 0.954 \\
\hline $\begin{array}{l}\text { White blood cell } \\
\text { count, } \times 10^{9} / \mathrm{L}\end{array}$ & $14.62 \pm 7.26$ & $15.61 \pm 7.42$ & $12.60 \pm 8.37$ & $14.22 \pm 7.23$ & $15.74 \pm 5.52$ & 1.479 & 0.222 \\
\hline Hemoglobin, $\mathrm{g} / \mathrm{L}$ & $113.77 \pm 17.91$ & $113.67 \pm 16.05$ & $113.12 \pm 18.04$ & $113.60 \pm 19.97$ & $114.82 \pm 18.04$ & 17.307 & 0.053 \\
\hline $\begin{array}{l}\text { Platelet count, } \\
\times 10^{9} / \mathrm{L}\end{array}$ & $405.96 \pm 187.33$ & $420.92 \pm 196.84$ & $386.42 \pm 193.32$ & $365.50 \pm 172.48$ & $462.15 \pm 178.91$ & 1.996 & 0.117 \\
\hline $\begin{array}{l}\text { Red blood cell } \\
\text { volume } \\
\text { distribution } \\
\text { width, \% }\end{array}$ & $14.70 \pm 1.92$ & $14.98 \pm 1.95$ & $14.94 \pm 2.32$ & $14.31 \pm 1.73$ & $14.59 \pm 1.68$ & 1.222 & 0.303 \\
\hline CRP, mg/L & $32.22(0.50-386.50)$ & $34.12(0.50-386.50)$ & $29.07(0.50-185.60)$ & $34.75(0.50-386.50)$ & $28.86(0.50-198.00)$ & 0.405 & 0.939 \\
\hline PCT, ng/L & $1.04(0.01-25.00)$ & $0.63(0.02-4.07)$ & $1.65(0.01-25.00)$ & $1.30(0.02-22.46)$ & $0.65(0.05-3.64)$ & 1.053 & 0.789 \\
\hline $\begin{array}{l}\text { Lactate } \\
\text { dehydrogenase, } \\
\text { U/L }\end{array}$ & $\begin{array}{c}810.11(136.80- \\
11000.00)\end{array}$ & $\begin{array}{l}612.50(158.80- \\
1740.00)\end{array}$ & $\begin{array}{c}1330.63(136.80- \\
11000.00)\end{array}$ & $\begin{array}{c}706.61(238.10- \\
2363.80)\end{array}$ & $\begin{array}{c}749.34(250.70- \\
4635.00)\end{array}$ & 1.788 & 0.617 \\
\hline $\begin{array}{l}\text { MB isoenzyme of } \\
\text { creatine kinase, } \\
\text { U/L }\end{array}$ & $46.23(3.00-300.00)$ & $41.31(6.00-203.00)$ & $57.47(10.30-295.20)$ & $37.06(3.00-300.00)$ & $55.63(5.00-202.50)$ & 5.975 & 0.113 \\
\hline $\begin{array}{l}\text { Alanine } \\
\text { aminotransferase, } \\
U / L\end{array}$ & $60.52(4.00-516.60)$ & $62.71(7.40-516.60)$ & $46.34(5.90-316.70)$ & $66.55(7.40-371.70)$ & $62.69(4.00-174.70)$ & 6.368 & 0.095 \\
\hline D-dimer, mg/L & $3.88(0.05-80.00)$ & $5.11(0.13-60.26)$ & $4.36(0.24-39.33)$ & $3.95(0.05-80.00)$ & $1.49(0.16-15.60)$ & 14.509 & 0.002 \\
\hline TLSH, d & $15.15(1.00-72.00)$ & $18.53(2.0-72.0)$ & $16.52(1.0-62.0)$ & $13.27(3.0-62.0)$ & $11.48(5.00-26.0)$ & 8.335 & 0.040 \\
\hline PRISM III scores & $2.85(0-33.00)$ & $4.51(0-32.00)$ & $4.15(0-33.00)$ & $1.63(0-16.00)$ & $0.88(0-5.00)$ & 151.243 & 0.000 \\
\hline $\begin{array}{l}\text { PRISM III } \\
\text { estimated } \\
\text { mortality (\%) }\end{array}$ & $3.24(0.41-88.22)$ & $5.00(0.55-84.84)$ & $5.28(0.41-88.22)$ & $1.43(0.49-18.60)$ & $1.21(0.65-10.15)$ & 29.495 & 0.000 \\
\hline CRP/PA & $2.40(0.02-39.36)$ & $3.69(0.05-39.36)$ & $2.35(0.03-16.11)$ & $1.99(0.02-19.84)$ & $1.12(0.02-7.18)$ & 8.038 & 0.045 \\
\hline PCT/PA & $0.08(0-2.71)$ & $0.07(0-0.37)$ & $0.14(0-2.17)$ & $0.08(0-1.47)$ & $0.02(0-0.13)$ & 8.982 & 0.030 \\
\hline
\end{tabular}

Abbreviations: CRP, C-reactive protein; PA, pre-albumin; PCT, procalcitonin; PRISM III, the pediatric risk of mortality III; TLSH, the total length of stay in hospital.

tolerance of the independent variables was greater than 0.2 , and the variance inflation factor of the independent variables was less than 10 , indicating that there was no collinearity among the independent variables (Table 3 ). The histogram of standardized residuals and the scatter plots of standardized residuals and predicted values suggested that the residuals passed the tests of normality and equal variance. The Durbin-Watson statistic was 2.107 (i.e., $\sim 2$ ) in the model summary form, so there was no signifi- cant correlation between the residuals (data not shown). Therefore, the multiple linear regression model fulfilled the five demands of linear correlation, no collinearity, normality and equal variance of residuals, and independent residuals, suggesting that the model was valid. From the multiple linear regression analysis model, the level of PA ( $\beta=-0.025,95 \%$ CI: -0.048 to $-0.002, \mathrm{P}=0.032$ ) was found to be negatively associated with the normal score of TLSH calculated using the Blom formula. 


\begin{tabular}{|c|c|c|c|c|c|c|c|c|}
\hline & \multirow{2}{*}{$\beta$} & \multirow{2}{*}{ S.E. } & \multirow{2}{*}{ Wals } & \multirow{2}{*}{ df } & \multirow{2}{*}{ sig } & \multirow{2}{*}{$\operatorname{Exp}(\beta)$} & \multicolumn{2}{|c|}{$\operatorname{Exp}(\beta) 95 \%$ Confidence Interval } \\
\hline & & & & & & & Lower Bound & Upper Bound \\
\hline Prealbumin & -0.168 & 0.046 & 13.163 & 1 & 0.000 & 0.846 & 0.773 & 0.926 \\
\hline D-dimer & 0.036 & 0.030 & 1.409 & 1 & 0.235 & 1.037 & 0.977 & 1.101 \\
\hline $\begin{array}{l}\text { Invasive } \\
\text { intubated }\end{array}$ & 1.522 & 0.497 & 9.379 & 1 & 0.002 & 4.581 & 1.730 & 12.124 \\
\hline TLSH & -0.003 & 0.016 & 0.028 & 1 & 0.868 & 0.997 & 0.966 & 1.030 \\
\hline $\mathbf{C R P} / \mathbf{P A}$ & 0.027 & 0.043 & 0.387 & 1 & 0.534 & 1.027 & 0.944 & 1.117 \\
\hline PCT/PA & -1.350 & 1.641 & 0.677 & 1 & 0.411 & 0.259 & 0.010 & 6.465 \\
\hline
\end{tabular}

Abbreviation: TLSH, the total length of stay in hospital

\begin{tabular}{|c|c|c|c|c|c|c|c|c|c|}
\hline \multirow{2}{*}{ Model } & \multicolumn{2}{|c|}{ Unstandardized Coefficients } & \multirow{2}{*}{$\begin{array}{c}\text { Standardized } \\
\text { Coefficients } \\
\text { Beta }\end{array}$} & \multirow{2}{*}{$\mathbf{t}$} & \multirow{2}{*}{ Sig. } & \multicolumn{2}{|c|}{ B 95\% Confidence Interval } & \multicolumn{2}{|c|}{ Collinearity Statistic } \\
\hline & $\beta$ & Std. Error & & & & $\begin{array}{l}\text { Lower } \\
\text { Bound }\end{array}$ & $\begin{array}{l}\text { Upper } \\
\text { Bound }\end{array}$ & Tolerance & VIF \\
\hline Prealbumin & -0.025 & 0.012 & 0.171 & -2.158 & 0.032 & -0.048 & -0.002 & 0.952 & 1.150 \\
\hline D-dimer & -0.010 & 0.009 & -0.088 & -1.080 & 0.282 & -0.028 & 0.008 & 0.897 & 1.115 \\
\hline $\begin{array}{l}\text { Invasive } \\
\text { intubated }\end{array}$ & 0.322 & 0.197 & 0.134 & 1.634 & 0.104 & -0.067 & 0.712 & 0.882 & 1.133 \\
\hline
\end{tabular}

${ }^{a}$ Dependent variable: Normal score of TLSH using blom's formula

\section{Discussion}

Serum PA, a negative acute-phase reactant, has been associated with neoplastic disease, acute kidney injury, brain injury, and cardiovascular diseases (15-18) Furthermore, many studies have shown the prognostic value of serum PA for infectious diseases. Cheng et al. reported that lower transthyretin (or PA) levels were associated with greater infectious complication rates, greater mortality, longer hospital stay, longer ICU stay, and increased ventilator days (19). Retrospective analysis indicated that PA in combination with the blood urea nitrogen concentration and the number of infected spaces can predict the length of hospital stay for patients with odontogenic infections (20). Salvetti et al. showed that in a consecutive series of 387 patients, the preoperative PA level correlated with the risk of surgical-site infection after elective spine surgery (21). Sun et al. also suggested that serum PA insufficiency is related to a greater recurrence risk for inflammatory processes (22). Ye et al. argued that the early measurement of serum PA during the acute phase of ischemic stroke may help identify patients at risk for strokeassociated infection, and may hence guide interventions to prevent stroke-associated infection (23). Other studies have indicated PA can be used as a reference marker to complement the chest X-rays for severity assessment of children CAP $(24,25)$. PA was useful for differentiating patients with refractory Mycoplasma pneumoniae pneumonia from those with the general Mycoplasma pneumoniae pneumonia (26). In this study, we found that lower PA levels were associated with increased PRISM III scores and prolonged TLSH, suggesting that PA may be involved in the progression of severe MPP and may contribute to its poor outcomes via mechanisms that remain to be clarified. Our findings are consistent with those of the above investigations. The following might explain our findings. First, severe MPP cascade releases a large number of inflammatory mediators and cytokines. The excess inflammatory mediators and cytokines interfere with hepatic protein synthesis and decrease the production of serum PA. The serum PA concentration falls in the presence of M. pneumoniae infection due to increased vascular permeability $(27,28)$. Second, critical illness (including severe MPP) is usually associated with a high catabolic state. Children in pediatric ICUs must be administered adequate nutrients to meet the increased dietary needs in critical illness, but this is not an easy task. PA levels are positively correlated with daily protein and energy intake, and the catabolic state associated with critical illness may lead to immunosuppression, poor wound healing, and ICU-acquired weakness, which in turn lead to increased mortality and delayed recovery (29). Third, low serum PA levels predict which critically ill patients may develop hypoglycemia and refeeding hy- 
pophosphatemia after at least $48 \mathrm{~h}$ of no nutrition, and severe hypoglycemia and hypophosphatemia confer a significant mortality risk on the hospitalized patient (30).

Because PA synthesis is influenced by inflammation, some studies have integrated inflammatory responses and nutrition status with their ratio $(12,31,32)$. to diminish their interference. A prospective cohort study of 70 critically ill patients demonstrated a strong correlation between the severity of organ dysfunction and the ratio of the two hepatic proteins CRP and PA(33). Another prospective study of 240 consecutive adult patients in a medical ICU showed that the CRP/PA ratio was independently correlated with hospital mortality and TLSH (34). Brown et al. (35) studied 20 trauma patients who received a highprotein enteral formulation within 5 days of injury. The serum PCT, CRP, and PA levels were analyzed on days 1 and 7 of enteral nutrition. The results suggested that the PCT/PA ratio had prognostic importance in determining clinical outcomes, especially in critically ill patients. In this study, we observed that the CRP/PA ratio gradually and significantly decreased as the serum PA level increased. Furthermore, the PCT/PA ratio significantly differed among the four PA groups. However, binary logistic regression analysis showed that the CRP/PA and PCT/PA ratios were not associated with PRISM III scores. These inconsistent results could be due to the limited sample size or due to regional and inter-individual differences between our study and the above studies.

This study has some limitations. First, the sample size of this study was small. Although selection bias is not entirely preventable, even in multicenter studies, further prospective studies may help to reduce this bias. Second, the results might be limited due to the fact that this study involved children who were admitted to a tertiary hospital, and therefore, patients with severe MPP may have been over-represented. Despite these limitations, our results provided useful insights into the correlation of serum PA levels with PRISM III scores and TLSH in children with MPP.

The present study demonstrated that low PA levels were associated with higher PRISM III scores and longer TLSH, suggesting that low PA levels contribute to the progression to severe MPP and possibly result in poor outcomes.

\section{Footnotes}

Authors' Contribution: Cheng-Yi Wang was responsible for the study concept and design, and writing of the manuscript. Chao-Min Song participated in the preliminary data collection and data analysis, and writing the manuscript. Guang-Hua Liu supervised the design and execution of the study. Shi-Biao Wang participated in patient care, data collection, and data analysis. All authors read and approved the final manuscript.

Clinical Trial Registration Code: ChiCTR1900023117.

Conflict of Interests: All authors declare that there are no conflicts of interest.

Ethical Approval: This study was approved by the Ethics Committee with No. 042 (2017).

Funding/Support: This work was supported by a grant from Open Fund of Engineering Research Center for Medical Data Mining and Application of Fujian Province (MDM2018004), the Scientific Research Foundation of Fujian Provincial Maternity and Children's Hospital (no, YCXZ18-08) and the Guiding Project of Science and Technology Department of Fujian Province (no, 2019Y0057).

\section{References}

1. Wang K, Gao M, Yang M, Meng F, Li D, Lu R, et al. Transcriptome analysis of bronchoalveolar lavage fluid from children with severe Mycoplasma pneumoniae pneumonia reveals novel gene expression and immunodeficiency. Hum Genomics. 2017;11(1):4. doi: 10.1186/s40246-017-0101-y. [PubMed: 28302172]. [PubMed Central: PMC5356355].

2. Lee H, Yun KW, Lee HJ, Choi EH. Antimicrobial therapy of macrolide-resistant Mycoplasma pneumoniae pneumonia in children. Expert Rev Anti Infect Ther. 2018;16(1):23-34. doi: 10.1080/14787210.2018.1414599. [PubMed: 29212389].

3. Colin AA, Yousef S, Forno E, Korppi M. Treatment of Mycoplasma pneumoniae in pediatric lower respiratory infection. Pediatrics. 2014;133(6):1124-5. doi: 10.1542/peds.2014-0871. [PubMed: 24864169].

4. Sawicki GS, Lu FL, Valim C, Cleveland RH, Colin AA. Necrotising pneumonia is an increasingly detected complication of pneumonia in children. Eur Respir J. 2008;31(6):1285-91. doi: 10.1183/09031936.00099807. [PubMed: 18216055].

5. Choi SY, Choi YI, Choi JH, Choi KD. Isolated optic neuritis associated with Mycoplasma pneumoniae infection: Report of two cases and literature review. Neurol Sci. 2017;38(7):1323-7. doi: 10.1007/s10072-0172922-9. [PubMed: 28321515].

6. Carrara C, Abbate M, Sabadini E, Remuzzi G. Acute kidney injury and hemolytic anemia secondary to Mycoplasma pneumoniae infection. Nephron. 2017;137(2):148-54. doi: 10.1159/000478991. [PubMed: 28683450].

7. Jin X, Zhu Y, Zhang Y, Chen J, Rong L, Zhao X. Assessment of levels of Ddimer and interferon-gamma in pediatric patients with Mycoplasma pneumoniae pneumonia and its clinical implication. Exp Ther Med. 2018;16(6):5025-30. doi: 10.3892/etm.2018.6873. [PubMed: 30546408]. [PubMed Central: PMC6256836].

8. Yan C, Sun H, Zhao H. Latest surveillance data on Mycoplasma pneumoniae infections in children, suggesting a new epidemic occurring in Beijing.JClin Microbiol.2016;54(5):1400-1. doi:10.1128/JCM.00184-16. [PubMed: 26912752]. [PubMed Central: PMC4844719].

9. Forga L, Bolado F, Goni MJ, Tamayo I, Ibanez B, Prieto C. Low serum levels of prealbumin, retinol binding protein, and retinol are frequent in adult type 1 diabetic patients. J Diabetes Res. 2016;2016:2532108. doi: 10.1155/2016/2532108. [PubMed: 28018921]. [PubMed Central: PMC5153501 publication of this paper].

10. Franco J, Formiga F, Trullas JC, Salamanca Bautista P, Conde A, Manzano L, et al. Impact of prealbumin on mortality and hospital readmission in patients with acute heart failure. Eur J Intern Med. 2017;43:36-41. doi: 10.1016/j.ejim.2017.05.009. [PubMed: 28499710]. 
11. Critsinelis AC, Kurihara C, Kawabori M, Sugiura T, Civitello AB, Morgan JA. Preoperative prealbumin level as a predictor of outcomes in patients who underwent left ventricular assist device implantation. Am J Cardiol. 2017;120(11):1998-2002. doi: 10.1016/j.amjcard.2017.08.004. [PubMed: 28958451].

12. Zhang C, Liu P, Xia K, Fang $\mathrm{H}$, Jiang $\mathrm{M}$, Xie Q, et al. Association of serum prealbumin with angiographic severity in patients with acute coronary syndrome. Med Sci Monit. 2017;23:40419. doi: 10.12659/msm.902348. [PubMed: 28827514]. [PubMed Central: PMC5574376].

13. Roche M, Law TY, Kurowicki J, Sodhi N, Rosas S, Elson L, et al. Albumin prealbumin, and transferrin may be predictive of wound complications following total knee arthroplasty. J Knee Surg. 2018;31(10):946-51. doi: 10.1055/s-0038-1672122. [PubMed: 30282102].

14. Rey C, Garcia-Hernandez I, Concha A, Martinez-Camblor P, Botran M, Medina A, et al. Pro-adrenomedullin, pro-endothelin-1, procalcitonin, C-reactive protein and mortality risk in critically ill children: A prospective study. Crit Care. 2013;17(5):R240. doi: 10.1186/cc13064. [PubMed: 24131732]. [PubMed Central: PMC3840693].

15. Zhang L, Chen QG, Li SQ, Zhang J, Min QH, Gao QF, et al. Preoperative fibrinogen to prealbumin ratio as a novel predictor for clinical outcome of hepatocellular carcinoma. Future Oncol. 2019;15(1):13-22. doi 10.2217/fon-2018-0376. [PubMed: 30139267].

16. You ZB, Lin KY, Zheng WP, Lin CJ, Lin F, Guo TL, et al. Association of prealbumin levels with contrast-induced acute kidney injury in elderly patients with elective percutaneous coronary intervention. Clin Interv Aging. 2018;13:641-9. doi: 10.2147/CIA.S162764. [PubMed: 29713148] [PubMed Central: PMC5909788].

17. Boselli M, Aquilani R, Baiardi P, Dioguardi FS, Guarnaschelli C, Achilli MP, et al. Supplementation of essential amino acids may reduce the occurrence of infections in rehabilitation patients with brain injury. Nutr Clin Pract. 2012;27(1):99-113. doi: 10.1177/0884533611431068. [PubMed: 22307494]

18. Wang W, Wang CS, Ren D, Li T, Yao HC, Ma SJ. Low serum prealbumin levels on admission can independently predict in-hospital adverse cardiac events in patients with acute coronary syndrome. Medicine (Baltimore). 2018;97(30). e11740. doi:10.1097/MD.0000000000011740. [PubMed: 30045342]. [PubMed Central: PMC6078736].

19. Cheng V, Inaba K, Haltmeier T, Gutierrez A, Siboni S, Benjamin E, et al. Serum transthyretin is a predictor of clinical outcomes in critically ill trauma patients. Surgery. 2015;158(2):438-44. doi: 10.1016/j.surg.2015.02.019. [PubMed: 26003908].

20. Cunningham LJ, Madsen MJ, Van Sickels JE. Using prealbumin as an inflammatory marker for patients with deep space infections of odontogenic origin. J Oral Maxillofac Surg. 2006;64(3):375-8. doi: 10.1016/j.joms.2005.11.008. [PubMed: 16487796].

21. Salvetti DJ, Tempel ZJ, Goldschmidt E, Colwell NA, Angriman F, Panczykowski DM, et al. Low preoperative serum prealbumin levels and the postoperative surgical site infection risk in elective spine surgery: A consecutive series. J Neurosurg Spine. 2018;29(5):549-52. doi: 10.3171/2018.3.SPINE171183. [PubMed: 30052149].

22. Sun J, Xiao Y, Zhang M, Ao T, Lang S, Wang J. Serum inflammatory markers in patients with adenovirus respiratory infection. Med Sci Monit. 2018;24:3848-55. doi: 10.12659/MSM.910692. [PubMed: 29877315]. [PubMed Central: PMC6020746].

23. Ye S, Lin SP, Wu K, Fan Y, Xu M. Serum prealbumin is a predictive biomarker for stroke-associated infection after an ischemic stroke. Int J Neurosci. 2017;127(7):601-5. doi: 10.1080/00207454.2016.1218874. [PubMed: 27476523].

24. Ning J, Shao X, Ma Y, Lv D. Valuable hematological indicators for the diagnosis and severity assessment of Chinese children with community-acquired pneumonia: Prealbumin. Medicine (Baltimore). 2016;95(47). e5452. doi: 10.1097/MD.0000000000005452. [PubMed: 27893691]. [PubMed Central: PMC5134884].

25. Xu JJ, Zhong HQ, Shu LH. Clinical value of serum albumin and prealbumin levels in children with lobar pneumonia caused by Mycoplasma pneumoniae. Int J Clin Exp Med. 2019;12(1):557-64.

26. Zhang Y, Zhou Y, Li S, Yang D, Wu X, Chen Z. The clinical characteristics and predictors of refractory Mycoplasma pneumoniae pneumonia in children. PLoS One. 2016;11(5). e0156465. doi: 10.1371/journal.pone.0156465. [PubMed: 27227519]. [PubMed Central: PMC4882022].

27. Huang HH, Hsu CW, Kang SP, Liu MY, Chang SJ. Association between illness severity and timing of initial enteral feeding in critically ill patients: A retrospective observational study. Nutr J. 2012;11:30. doi: 10.1186/1475-2891-11-30. [PubMed: 22554240]. [PubMed Central: PMC3436719].

28. Malave I, Vethencourt MA, Pirela M, Cordero R. Serum levels of thyroxine-binding prealbumin, C-reactive protein and interleukin6 in protein-energy undernourished children and normal controls without or with associated clinical infections. J Trop Pediatr. 1998;44(5):256-62. doi: 10.1093/tropej/44.5.256. [PubMed: 9819485].

29. Arabi YM, Al-Dorzi HM, Mehta S, Tamim HM, Haddad SH, Jones G, et al. Association of protein intake with the outcomes of critically ill patients: A post hoc analysis of the PermiT trial. Am J Clin Nutr. 2018;108(5):988-96. doi: 10.1093/ajcn/nqy189. [PubMed: 30475959].

30. Gaudiani JL, Sabel AL, Mehler PS. Low prealbumin is a significant predictor of medical complications in severe anorexia nervosa. Int J Eat Disord. 2014;47(2):148-56. doi: 10.1002/eat.22233. [PubMed: 24375513].

31. Lu J, Xu BB, Zheng ZF, Xie JW, Wang JB, Lin JX, et al. CRP/prealbumin, a novel inflammatory index for predicting recurrence after radical resection in gastric cancer patients: post hoc analysis of a randomized phase III trial. Gastric Cancer. 2019;22(3):536-45. doi: 10.1007/s10120018-0892-0. [PubMed: 30377862].

32. Davis CJ, Sowa D, Keim KS, Kinnare K, Peterson S. The use of prealbumin and C-reactive protein for monitoring nutrition support in adult patients receiving enteral nutrition in an urban medical center. JPEN J Parenter Enteral Nutr. 2012;36(2):197-204. doi: 10.1177/0148607111413896. [PubMed: 21799187].

33. Pinilla IC, Hayes P, Laverty W, Arnold C, Laxdal V. The C-reactive protein to prealbumin ratio correlates with the severity of multiple organ dysfunction. Surgery. 1998;124(4):799-805. discussion 805-6. doi: 10.1067/msy.1998.91365. [PubMed: 9781004].

34. Li L, Dai L, Wang X, Wang Y, Zhou L, Chen M, et al. Predictive value of the C-reactive protein-to-prealbumin ratio in medical ICU patients. BiomarkMed.2017;11(4):329-37. doi:10.2217/bmm-2016-0266. [PubMed: 28326826].

35. Brown RO, Alexander EJ, Hanes SD, Wood GC, Kudsk KA, Dickerson RN. Procalcitonin and enteral nutrition tolerance in critically ill patients. JPEN J Parenter Enteral Nutr. 2003;27(1):84-8. doi: 10.1177/014860710302700184. [PubMed: 12549604] 